\title{
A Closed-Cycle Miniature Dilution Refrigerator for a Fast-Cooldown 100 mK Detector Wafer Test Cryostat
}

\author{
S. Azzoni ${ }^{1,2}$ (D) A. J. May ${ }^{1,3,4}$ - S. T. Chase ${ }^{5}$ G. Coppi ${ }^{6}$ - L. C. Kenny ${ }^{5}$. \\ S. J. Melhuish ${ }^{1} \cdot$ L. Piccirillo ${ }^{1,7} \cdot$ A. Suzuki ${ }^{8} \cdot$ J. Wenninger ${ }^{1,2}$
}

Received: 20 August 2019 / Accepted: 30 January 2020 / Published online: 24 February 2020

(c) The Author(s) 2020

\begin{abstract}
The forthcoming generation of cosmic microwave background polarization observatories is developing large format detector arrays which will operate at $100 \mathrm{mK}$. Given the volume of detector wafers that will be required, fast-cooldown $100 \mathrm{mK}$ test cryostats are increasingly needed. A miniature dilution refrigerator (MDR) has been developed for this purpose and is reported. The MDR is precooled by a doublestage ${ }^{3} \mathrm{He}-{ }^{4} \mathrm{He}$ Chase Research Cryogenics sorption refrigerator. The test cryostat based on this MDR will enable fast cooldown to $100 \mathrm{mK}$ to support rapid feedback testing of detector wafers fabricated for the Simons Observatory. The MDR has been designed to provide a $100 \mathrm{mK}$ stage to be retrocompatible with existing CRC10 sorption coolers, reducing the base temperature from $250 \mathrm{mK}$ for the new generation of detectors. Other $250 \mathrm{mK}$ cryostats can be retrofitted in the same way. This configuration will meet the cryogenic requirements for single-wafer testing, providing $5-10 \mu \mathrm{W}$ of cooling power at $100 \mathrm{mk}$ for over $8 \mathrm{~h}$. The system operates in a closed cycle, thereby avoiding external gas connections and cold o-rings. No moving parts are required, with the system operated entirely by heaters.
\end{abstract}

Keywords Dilution · Cryogenics · Cosmic microwave background · Instrumentation

\section{Introduction}

The forthcoming generation of cosmic microwave background observatories will target an ambitious set of science goals requiring unprecedented sensitivities. To support this, the detector count will increase by approximately an order of magnitude and focal planes will operate at temperatures $\sim 100 \mathrm{mK}$. The scaling up of fabrication of highperformance low-temperature detector wafers requires timely feedback on the yield and initial performance. This justifies the need to rely on test bench with prompt feedback

S. Azzoni

susanna.azzoni@postgrad.manchester.ac.uk

Extended author information available on the last page of the article 


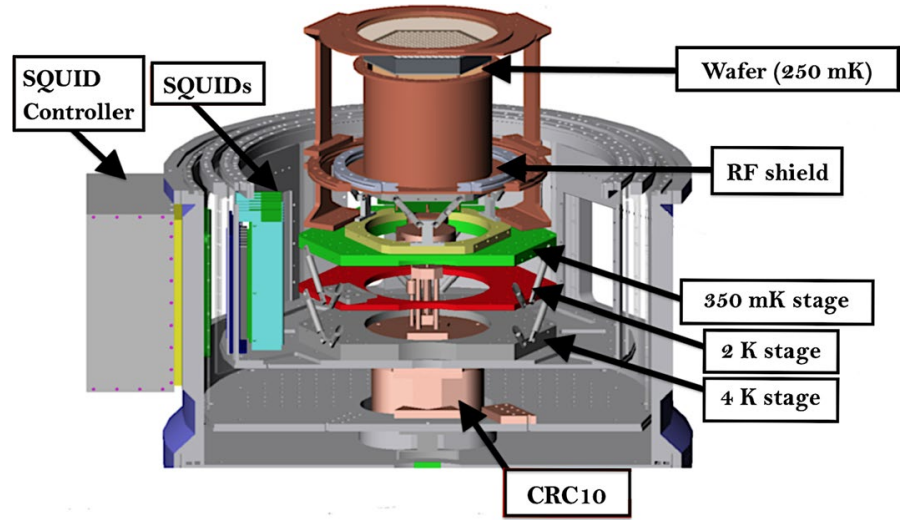

Fig. 1 Cutout schematics of the existing APEX test cryostat at UC Berkeley (adapted from [14]) (Color figure online)

testing capabilities operating at $100 \mathrm{mK}$ [1]. For this purpose, a test cryostat based on a novel miniature dilution refrigerator (MDR) has been developed [2].

Several MDR designs have been reported [3-10]. In condensation pumped MDRs, a small amount of ${ }^{3} \mathrm{He}$ circulates within the cold stage, offering several advantages over conventional systems. Crucially, since they do not need room temperature gas circulation, the smaller-scale pumping lines considerably reduce size and complexity of the overall system. The system operates in a closed cycle, thereby avoiding cold o-rings and external gas handling systems, which require high power consumption and may be prohibitively expensive, mostly due to the required amount of ${ }^{3} \mathrm{He}$. No moving parts are required, with the system operated entirely by heaters. It is possible to fully automate the cycling of each stage in order to provide "pushbutton" cooldown to below $100 \mathrm{mK}$.

A design has been developed for such a system that can be easily mounted on existing ${ }^{4} \mathrm{He}-{ }^{3} \mathrm{He}-{ }^{3} \mathrm{He}$ sorption coolers. This is capable of meeting the cooling power requirements for preliminary testing at $100 \mathrm{mK}$ of detector wafers fabricated for the Simons Observatory [11]. The design modifies the existing APEX-SZ [12] cryostat at UC Berkeley which is currently being used for POLARBEAR-2 [13] detector testing. Moreover, the architecture of the system that has been developed is convenient since it could easily be implemented for other low-temperature detector applications requiring comparable cooling powers at $\sim 100 \mathrm{mK}$.

\section{Detector Test Cryostat Development}

The APEX-SZ cryostat is shown in Fig. 1. Coaxial $40 \mathrm{~K}$ and $4 \mathrm{~K}$ stages are cooled by a pulse tube cryocooler. A three-stage, ${ }^{4} \mathrm{He}-{ }^{3} \mathrm{He}-{ }^{3} \mathrm{He}$ ("He-10") sorption refrigerator provided by Chase Research Cryogenics (CRC) ${ }^{1}$ cools the focal plane

\footnotetext{
1 www.chasecryogenics.com.
} 
to $\sim 250 \mathrm{mK}$, at which stage the detector wafer under test is placed. The CRC10 cooler uses the ${ }^{4} \mathrm{He}$ stage to precool the first ${ }^{3} \mathrm{He}$ coldhead to $1 \mathrm{~K}$, with a $2 \mathrm{~K}$ stage provided by a film breaker. The film breaker prevents the development of ${ }^{4} \mathrm{He}$ film due to the thermomechanical effect and is positioned at the top of the evaporator to minimize the heat leak from the $4 \mathrm{~K}$ stage. A film breaker of similar operation is described in detail in Ref. [15]. Once the ${ }^{4} \mathrm{He}$ charge has expired, the first ${ }^{3} \mathrm{He}$ pump is cooled, pumping the condensed ${ }^{3} \mathrm{He}$ in the ${ }^{3} \mathrm{He}$ coldhead down to $350 \mathrm{mK}$. This stage also provides further precooling and thermal intercept for the second ${ }^{3} \mathrm{He}$ stage which is then pumped down to $250 \mathrm{mK}$, cooling the detector wafer. In order to provide a $100 \mathrm{mK}$ base temperature, the second ${ }^{3} \mathrm{He}$ stage, or ultracold (UC) stage, is replaced with a MDR, which is then cooled by the first ${ }^{3} \mathrm{He}$ stage, or inter-cooler (IC) stage. This simple design is compatible with the architecture of the CRC10 and is described in the next paragraph.

\section{Test bed Cryostat Architecture}

A dry (cryogen-free) cryostat architecture is used. The test bed cryostat is precooled by a standard two-stage Gifford-McMahon cryocooler (GM). Each stage has an associated radiation shield and a copper plate, providing isothermal surfaces at $\sim 40 \mathrm{~K}$ and $\sim 4 \mathrm{~K}$. The type of GM used, Sumitomo RDK-415D, ${ }^{2}$ has a nominal cooling power of $1.5 \mathrm{~W}$ at $4 \mathrm{~K}$.

The double-stage ${ }^{3} \mathrm{He}-{ }^{4} \mathrm{He}$ sorption cooler (CRC7) precools the MDR and is mounted underneath the GM $4 \mathrm{~K}$ plate vertically with the coldhead downwards, using five OFHC struts. In order to minimize the turnaround time for detector wafer testing, the precooling stages are cycled automatically by an XML script which controls the various heaters in the system. As such, a wafer may be mounted in the test cryostat, the system is closed and evacuated, and the cryocooler and scripts run overnight to reach $100 \mathrm{mK}$ for testing the following morning.

\section{Precooling Stage to $350 \mathrm{mK}$}

In a CRC10 cooler with the UC stage removed, the ${ }^{3} \mathrm{He}$ and ${ }^{4} \mathrm{He}$ charcoal-loaded cryopumps are operated by heaters and gas-gap heat switches. The switches are mounted on top of the unit main plate and are thermally connected to the top of each cryopump by means of a heat strap. A pumping line runs from the cryopumps down to a condenser block, which is connected by thin-walled stainless steel tubes to an evaporator. A system schematic is shown in Fig. 2. The cryopumps are used to pump on the ${ }^{3} \mathrm{He}$ and ${ }^{4} \mathrm{He}$ coaxial pots located in the coldhead. While the heat switches are closed, the pumps reach thermal equilibrium with the $4 \mathrm{~K}$ stage. At $4 \mathrm{~K}$, the charcoal adsorbs the helium gas, acting as a very effective pump. Subsequently opening the heat switches and heating the pumps to $40 \mathrm{~K}$ cause the charcoal to desorb the

\footnotetext{
${ }^{2}$ www.shicryogenics.com.
} 


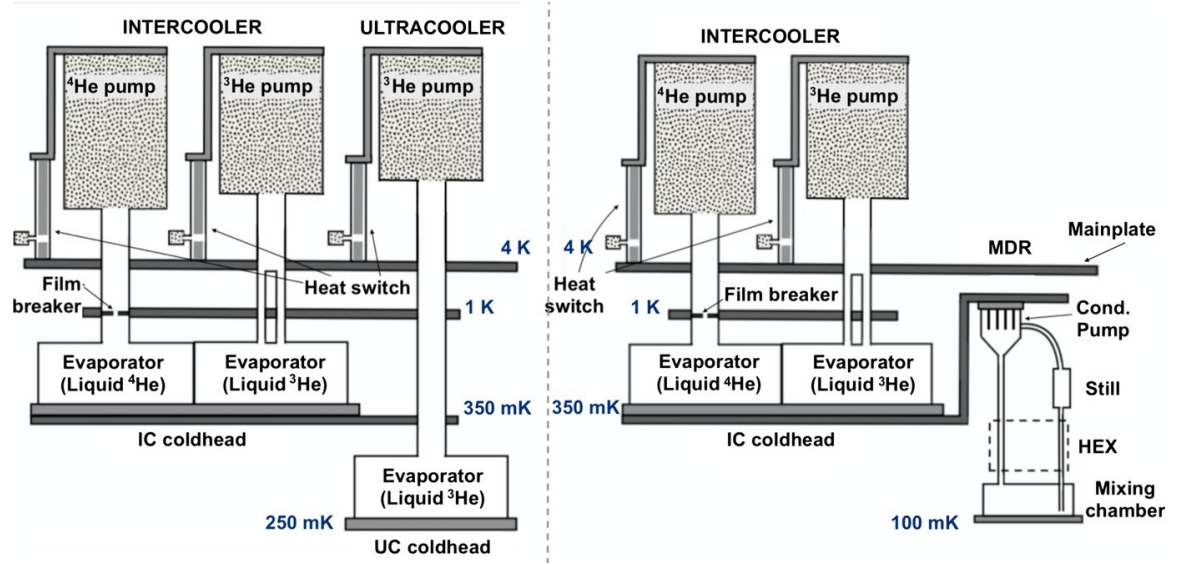

Fig. 2 Left: Schematic figure of the ${ }^{4} \mathrm{He}-{ }^{3} \mathrm{He}-{ }^{3} \mathrm{He}$ (CRC10) fridge. Right: Schematic figure of the ${ }^{4} \mathrm{He}-{ }^{3} \mathrm{He}$ (CRC7) fridge and MDR. The ultracooler stage has been replaced with a MDR to reduce the base temperature to $100 \mathrm{mK}$ (Color figure online)
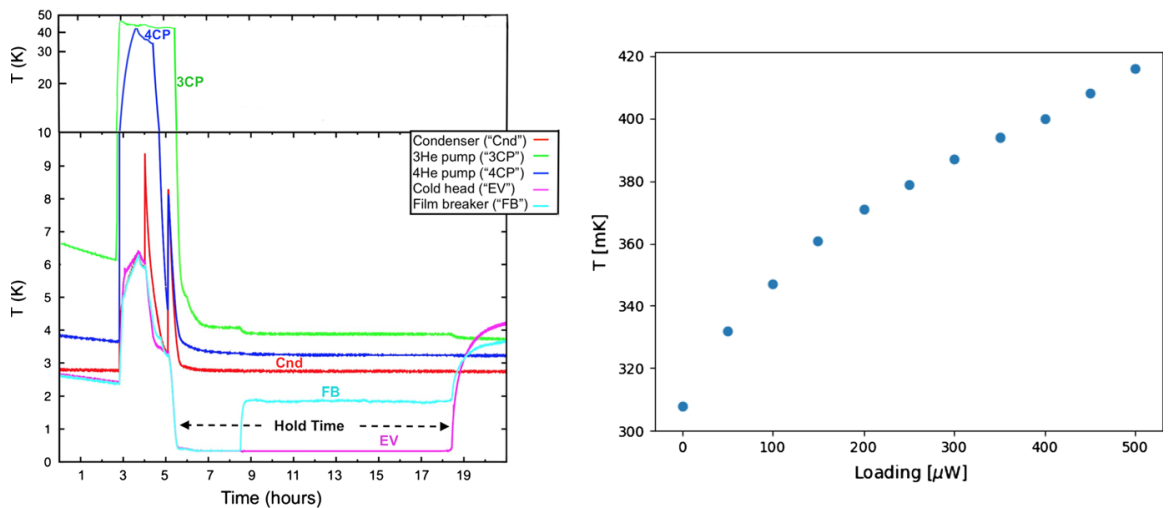

Fig. 3 Left: Cycle procedure of the cooler. The figure shows the temperature of each component over time. Right: Load curve measured for the IC stage (Color figure online)

helium gas [16] which condenses and collects as liquid in the coldhead. Keeping the ${ }^{3} \mathrm{He}$ pump warm, the ${ }^{4} \mathrm{He}$ pump heater is switched off and the corresponding switch is closed. As a result, the cryopump cools down and pumps on the liquid in the ${ }^{4} \mathrm{He}$ pot, reducing the temperature of the coldhead. Once the ${ }^{4} \mathrm{He}$ is exhausted, the ${ }^{3} \mathrm{He}$ pump heater is switched off and the switch closed, pumping the ${ }^{3} \mathrm{He}$ down to its base temperature $\sim 310 \mathrm{mK}$.

An example of a cooling cycle is shown in Fig. 3. The cycle has been repeated several times and showed that the refrigerator maintains a temperature $\sim 380 \mathrm{mK}$ for over $12 \mathrm{~h}$, under an artificial load of $\sim 330 \mu \mathrm{W}$ provided by a heater mounted to the coldhead. Once ${ }^{3} \mathrm{He}$ is completely exhausted, the evaporator will begin to warm and the system needs to be recycled. It took $\sim 3 \mathrm{~h}$ to recycle the system and bring it back to its 


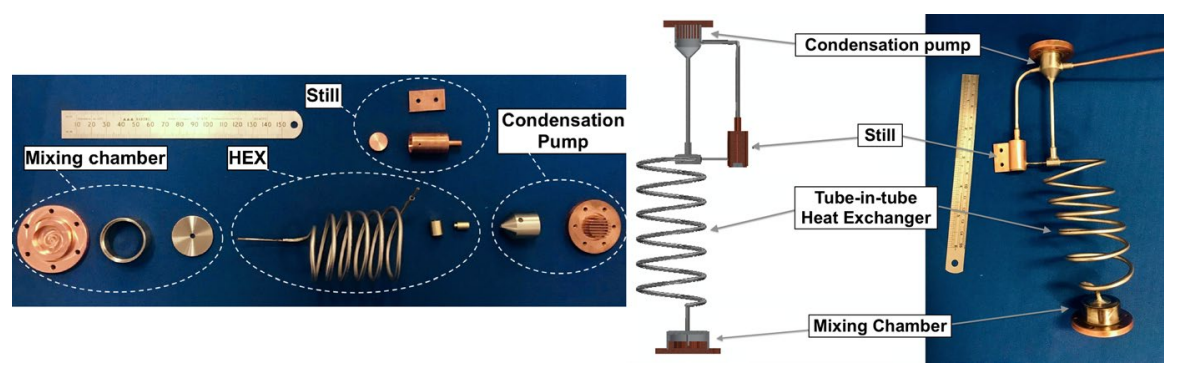

Fig. 4 Left: Manufactured parts before assembly. Right: Cutaway 3D CAD rendering and assembled preliminary MDR prototype (Color figure online)

base temperature. One-third of the recycle time was used to allow the GM to recover from the heating of the cryopumps, which ensured an efficient condensation of ${ }^{4} \mathrm{He}$. A more powerful cryocooler may be used to reduce the recycle time.

\section{Miniature Dilution Refrigerator}

The test of the detector requires an operating temperature of $100 \mathrm{mK}$ for more than $8 \mathrm{~h}$, keeping an heat load higher than $5 \mu \mathrm{W}$ at $100 \mathrm{mK}$. A prototype cooler, which is expected to meet the cooling power and hold time requirements, has been manufactured, as shown in Fig. 4 with an illustrative schematic in Fig. 2.

\subsection{Design}

A condensation pump chamber at the top is mounted to the IC coldhead via a thermal link. A return line connects the bottom of this chamber with the mixing chamber. A second tube runs from the bottom of the mixing chamber up to the still through a heat exchanger assembly. Another tube then runs from the top of the still back to the condensation pump to allow pumping of the ${ }^{3} \mathrm{He}$ gas.

While ${ }^{3} \mathrm{He}$ flows from the still, cooling at $100 \mathrm{mK}$ is supplied to the detector wafer under test. The cooling power of the dilution process is given by the difference in enthalpy between the helium leaving and entering the chamber. In this temperature regime, the molar enthalpy of the dilute solution and that of the incoming pure ${ }^{3} \mathrm{He}$ approximate to $94 T^{2} \mathrm{~J} / \mathrm{mol}$ and $12 T^{2}$, respectively [17]; hence, the cooling power of the MDR is

$$
\dot{Q}_{\mathrm{MDR}}=\dot{n}\left(94 T_{\mathrm{m}}^{2}-12 T_{\mathrm{i}}^{2}\right)
$$

where $\dot{n}$ is the molar flow rate of ${ }^{3} \mathrm{He}, T_{\mathrm{m}}$ is the temperature of the mixing chamber and $T_{\mathrm{i}}$ is the temperature of the returning ${ }^{3} \mathrm{He}$. The maximum cooling power is achieved if a perfect heat exchanger is assumed, such that $T_{\mathrm{i}}=T_{\mathrm{m}}$. Given the heat lift and temperature requirements, Eq. 1 corresponds to a flow rate $\dot{n} \sim 6 \mu \mathrm{mol} / \mathrm{s}$. The minimum temperature reached at the mixing chamber is therefore limited by the 
heat load on the $350 \mathrm{mK}$ stage, the helium circulation rate within the MDR, the heat exchanger efficiency and the Kapitza resistance. The ${ }^{3} \mathrm{He}$ circulation rate is determined by the pumping rate from the still to the condensation pump. That is, keeping the temperature of the still well above that of the condensation pump improves its efficiency. Applying power to the still raises the still temperature up to a limit set by the capacity of the condensation pump. The heat load on the condensation pump is $\dot{n} \Delta H$, where $\Delta H$ is the change in enthalpy leaving and entering the pump. After being removed from the still, ${ }^{3} \mathrm{He}$ cools down from the still temperature to the temperature of the condensation pump, where it is pulled down by gravity as liquid. Therefore, $\Delta H$ is given by the difference in enthalpy entering and leaving the still in addition to the latent heat of evaporation, $L$, which is the dominating term. Hence, the flow rate is

$$
\dot{n} \sim \frac{\dot{Q}_{\mathrm{S}}}{L}
$$

At a still temperature $700 \mathrm{mK}, L \sim 24 \mathrm{~J} / \mathrm{mol}$ [17]. Hence, a flow rate of $6 \mu \mathrm{mol} / \mathrm{s}$ corresponds to a still power $\dot{Q}_{\mathrm{S}}=144 \mu \mathrm{W}$ (assuming an ideal heat exchanger). The measured load curve in Fig. 3 shows that this results in a temperature of the condensation pump of $\sim 360 \mathrm{mK}$.

However, ideal heat exchanger performance will clearly not be attained in reality due to the contribution of several heat leaks. CuNi tube-in-tube continuous counterflow heat exchangers have been shown to be well suited to DRs with flow rates on the order of a few $\mu \mathrm{mol} / \mathrm{s}$ with $100 \mathrm{mK}$ base temperatures [9]. Several models [9, 18-20] estimated the efficiency of the heat exchanger as

$$
\frac{\dot{Q}}{\dot{n}}=\frac{\left(\gamma_{D}-\gamma_{C}\right)\left[\gamma_{D}(f-1)-\gamma_{C}\right]}{\gamma_{D}(f-1)-\gamma_{C}(f+1)}
$$

where the enthalpy coefficients of the diluted and the concentrated phase of the ${ }^{3} \mathrm{He} /{ }^{4} \mathrm{He}$ mixture are $\gamma_{D}=107 \mathrm{~J} / \mathrm{K}^{2} / \mathrm{mol}$ and $\gamma_{C}=23 \mathrm{~J} / \mathrm{K}^{2} / \mathrm{mol}$ [9], respectively, and $f$ is a dimensionless factor given by

$$
f=\exp \left(\frac{A / a_{k}}{\dot{n} \gamma_{D} \gamma_{C}}\left[\left(\gamma_{D}-\gamma_{C}\right) T_{m}^{2}-\frac{2 \dot{Q}}{\dot{n}}\right]\right)
$$

where $A$ is the surface area of the heat exchanger inner tube, $a_{K}$ is the Kapitza resistivity and $T_{m}$ is the MDR lowest achievable temperature. The area $A>40 \mathrm{~cm}^{2}$ has been chosen to suit the cooling power requirements, in order to minimize the effect of Kapitza resistance. Furthermore, the resistivity is the same on the concentrated side and the diluted side [20]; therefore, $a_{K}$ is taken to be $0.02 \mathrm{~m}^{2} \mathrm{~K}^{4} / \mathrm{W}$, twice the value of $a_{K}$ between diluted ${ }^{3} \mathrm{He}$ and $\mathrm{CuNi}$ [7].

\subsection{Preliminary Testing and Discussion}

The mixing chamber requires precooling to $<500 \mathrm{mK}$ in order to induce phase separation and operate the MDR. The dilution unit has been tested in this configuration. 

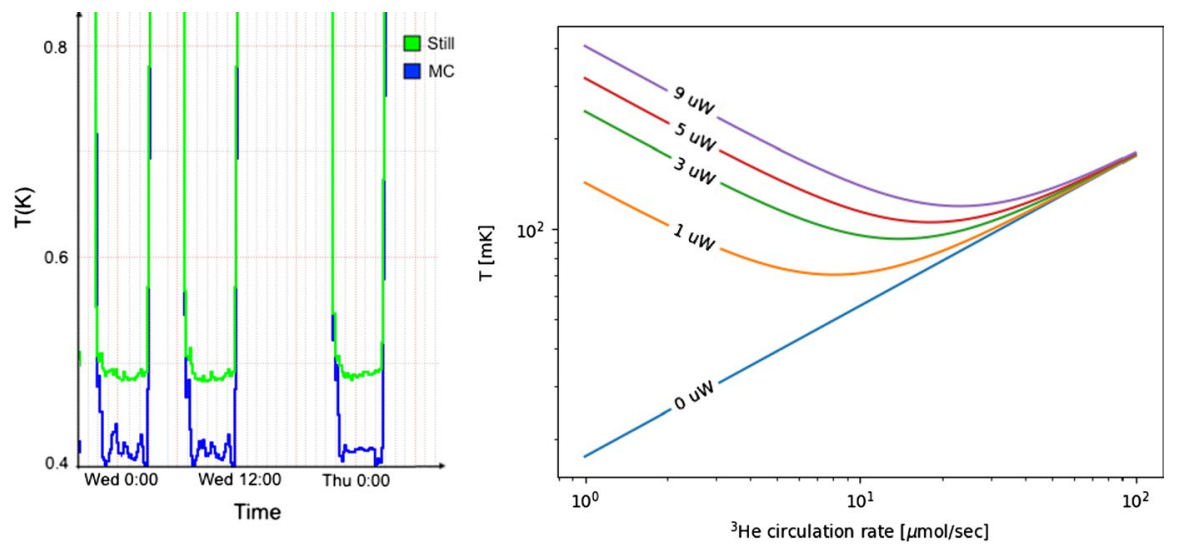

Fig. 5 Left: MDR dilution runs, showing the temperature in Kelvin of the MDR stages (still and mixing chamber) over time. Right: MDR mixing chamber lowest achievable temperature as a function of the circulation rate for different residual heat leaks (Color figure online)

However, several improvements to the preliminary design were necessary to activate dilution. In order to precool the mixing chamber to $4 \mathrm{~K}$ and then to $500 \mathrm{mK}$ while cycling the IC stage, an active ${ }^{3} \mathrm{He}$ gas-gap switch has been introduced to couple the IC head and the mixing chamber. Since the density ratio between ${ }^{4} \mathrm{He}$ and ${ }^{3} \mathrm{He}$ is $\rho_{4} / \rho_{3}=1.7$, the phase separation must be such that the still side of the circuit contains dilute mixture and the condenser side contains concentrate, while the MDR is in operation. To this end, there must be a difference in the column height in the equilibrium condition. Therefore, the original design of the MDR has been modified to include a higher mixing chamber with a total volume $7 \mathrm{~cm}^{3}$. In order to maintain a fixed $180 \mathrm{~mm}$ total height of the MDR unit, the HEX has been further compressed and aligned with the condenser. The still of volume $1.5 \mathrm{~cm}^{3}$ has been positioned approximately halfway between the mixing chamber and the condenser.

Taking into account the HEX, the total volume of the MDR amounts to $10 \mathrm{~cm}^{3}$. An external gas bottle connected to the MDR circuit has been charged with $7 \mathrm{~L}$ (STP) with a mixture of ${ }^{3} \mathrm{He}$ (4 L STP) and ${ }^{4} \mathrm{He}$ (3 L STP).

In order to fully charge the MDR with the overall amount of mixture, the CRC7 was cycled with the precooling switch closed. The precooling unit has been cycled multiple times, and the active switch successfully precooled the MC to $400 \mathrm{mK}$ at which point phase separation occurred, as shown in Fig. 5.

Nonetheless, while trying to artificially load the still, the temperature would not reach the $700 \mathrm{mK}$ expected from a loading $>144 \mu \mathrm{W}$ to allow an efficient performance of the dilutor. In fact, not all of the mixture has been successfully introduced in the dilution unit due to a slow transfer rate, leaving the still and the MC partially empty. Most of the mixture was drawn into the MDR, while the MC was precooled to the $\mathrm{CRC} 7$ base temperature, which shows that the mixture was not condensing well at $4 \mathrm{~K}$. Therefore, a condenser at the connection between the MDR and the $4 \mathrm{~K}$ stage will be added. Testing of the improved prototype has not yet been completed; however, results with the former prototype demonstrated 
that the MDR is mechanically and structurally functional. Furthermore, Eq. 4 has been used to estimate $T_{m}$ for different heat leaks in Fig. 5, corresponding to the contributions from viscous heating and lateral heat conduction in the heat exchanger. Given the above considerations and the measured load curve in Fig. 3, the system will comfortably support a circulation rate of $5<\dot{n}<11 \mu \mathrm{mol} \cdot \mathrm{s}^{-1}$, affording a maximum heat load of $10 \mu \mathrm{W}$ at the mixing chamber $(100 \mathrm{mK})$.

\section{Conclusion}

A sorption-cooled miniature dilution refrigerator has been developed for a test cryostat to provide rapid cooldown and support preliminary tests of detectors operating at $100 \mathrm{mK}$. The design, operation and thermodynamic analysis have been described. The proposed design meets the criteria for several low-temperature detector experiments and will offer a considerable advantage over existing three-stage sorption coolers which operate at $250 \mathrm{mK}$. The sorption cooler in the precooling stage has been operated extensively, and its performance has been reported. A prototype MDR has been manufactured and tested, showing a successful phase separation at the mixing chamber; further experimental tests are currently taking place and the authors look forward to reporting full experimental results in due course.

Open Access This article is licensed under a Creative Commons Attribution 4.0 International License, which permits use, sharing, adaptation, distribution and reproduction in any medium or format, as long as you give appropriate credit to the original author(s) and the source, provide a link to the Creative Commons licence, and indicate if changes were made. The images or other third party material in this article are included in the article's Creative Commons licence, unless indicated otherwise in a credit line to the material. If material is not included in the article's Creative Commons licence and your intended use is not permitted by statutory regulation or exceeds the permitted use, you will need to obtain permission directly from the copyright holder. To view a copy of this licence, visit http://creativecommons.org/licen ses/by/4.0/.

\section{References}

1. M. Abitbol et al., arXiv:1706.02464v2 (2017)

2. A. May, Sub-Kelvin Cryogenics for Experimental Cosmology, Ph.D. thesis, University of Manchester (2019)

3. A. May, S. Azzoni et al., IOP Conf. Ser.: Mater. Sci. Eng. 502, (2019)

4. A. Paiella et al., JCAP 2019, 039 (2019)

5. L. Piccirillo, G. Coppi, A. May, Miniature Sorption Coolers: Theory and Applications (CRC Press, Boca Raton, 2018)

6. S. Melhuish, L. Martinis, L. Piccirillo, J. Cryog. 55, 63 (2013). https://doi.org/10.1016/j.cryog enics.2013.03.002

7. V. Sivokon et al., Cryogenics 32, 207 (1992). https://doi.org/10.1016/0011-2275(92)90144-Y

8. V. Edel'man, Cryogenics 12, 385 (1972). https://doi.org/10.1016/0011-2275(72)90114-2

9. G. Teleberg, S. Chase, L. Piccirillo, J. Low Temp. Phys. 151, 669 (2008). https://doi.org/10.1007/ s10909-008-9724-7

10. T. Prouvé et al., Proceedings of the 15th International Cryocooler Conference, (2008)

11. N. Galitzki et al., Proc. SPIE 10708, 1070804 (2018). https://doi.org/10.1117/12.2312985 
12. M. Dobbs et al., New Astron. Rev. 50, 960 (2006). https://doi.org/10.1016/j.newar.2006.09.029

13. A. Suzuki et al., J. Low Temp. Phys. 184, 805 (2016). https://doi.org/10.1007/s10909-015-1425-4

14. A. Suzuki, Multichroic Bolometric Detector Architecture for Cosmic Microwave Background Polarimetry Experiments, Ph.D. thesis, UC Berkeley (2013)

15. A. May et al., Cryogenics 102, 45 (2019)

16. F. Pobell, Matter and Methods at Low Temperatures (Springer, New York, 1996)

17. R. Radebaugh, NBS Technical Note 392, (1967)

18. G. Frossati et al., Proceedings of the ULT Hakoné Symposium, Japan (1977), p.205

19. G. Frossati, J. Low Temp. Phys. (1992). https://doi.org/10.1007/BF00114918

20. Y. Takano, Rev. Sci. Instrum. (1994). https://doi.org/10.1063/1.1144857

Publisher's Note Springer Nature remains neutral with regard to jurisdictional claims in published maps and institutional affiliations.

\section{Affiliations}

\section{S. Azzoni ${ }^{1,2}$ (1) - A. J. May ${ }^{1,3,4}$. S. T. Chase ${ }^{5}$. G. Coppi ${ }^{6}$. L. C. Kenny ${ }^{5}$.}

\section{S. J. Melhuish ${ }^{1} \cdot$ L. Piccirillo ${ }^{1,7} \cdot$ A. Suzuki ${ }^{8} \cdot$ J. Wenninger ${ }^{1,2}$}

1 Jodrell Bank Centre for Astrophysics, School of Physics and Astronomy, University of Manchester, Oxford Road, Manchester M13 9PL, UK

2 Department of Astrophysics, University of Oxford, Denys Wilkinson Building, Keble Road, Oxford OX1 3RH, UK

3 RF and Cryogenics Group, Accelerator Science and Technology Centre, STFC Daresbury Laboratory, Keckwick Lane, Warrington WA4 4AD, UK

4 Cockcroft Institute, STFC Daresbury Laboratory, Keckwick Lane, Warrington WA4 4AD, UK

5 Chase Research Cryogenics Ltd., Cool Works, Unit 2 Neepsend Ind Est, Parkwood Road, Sheffield S3 8AG, UK

6 Department of Physics and Astronomy, University of Pennsylvania, 209 South 33rd Street, Philadelphia, PA 19104, USA

7 Engineering Research Institute Ventspils International Radio Astronomy Centre (ERI VIRAC) of Ventspils, University of Applied Sciences (VUAS), Ventspils 3601, Latvia

8 Physics Division, Lawrence Berkeley National Laboratory, Berkeley, 1-Cyclotron Road, CA 94720, USA 